\section{Exactitud de tamizaje de retinopatía diabética: inteligencia artificial versus tecnólogos médicos entrenados}

\author{
MARÍA C. IBÁÑEZ-BRURON ${ }^{1, \mathrm{a}, \mathrm{b}}$, ANDREA CRUZAT ${ }^{1,2}$, \\ GONZALO ÓRDENES-CAVIERES ${ }^{1, \mathrm{~b}}$, MARCELO CORIA $^{2}$
}

\section{Accuracy of artificial intelligence compared to trained medical technologists in diabetic retinopathy screening}

Background: The early detection of retinopathy among diabetics is of utmost importance. Aim: To estimate the diagnostic accuracy of two diabetic retinopathy (DR) screening strategies currently used in the Chilean public health system. Material and Methods: Cross-sectional observational study of 371 diabetic patients aged $61 \pm 14$ years ( $61 \%$ women) who underwent DR screening at a public Hospital between July 1 and August 31, 2019. The mydriatic retinal photographs of all participants were classified using artificial intelligence software (DART) and trained medical technologists, independently. The precision of both strategies was compared with the reference standard, namely the evaluation of the fundus by an ophthalmologist with a slit lamp. Participants with severe non-proliferative $D R$ or worse were considered as positive cases. The ophthalmologist was blind to the results of the screening tests. Results: Twenty four percent of participants had $D R$, including 34 (9.2\%) who had sight threatening DR in at least one eye. The sensitivity and specificity of DART were 100\% (95\% confidence intervals (CI): 90-100\%) and 55,4\% (95\% CI: 50-61\%), respectively. Medical technologists had a sensitivity of $97,1 \%$ (95\% CI: 85-100\%) and a specificity of $91,7 \%$ (95\% CI: 88-94\%). The only case missed by medical technologists was a patient with unilateral panphotocoagulated DR. Conclusions: Both strategies had a similar sensitivity to detect cases of sight-threatening DR. However, the specificity of $D A R T$ was significantly lower compared to medical technologists, which would greatly increase the burden on the health system, a very important aspect to consider in a screening strategy.

(Rev Med Chile 2021; 149: 493-500)

Key words: Artificial Intelligence; Diabetic Retinopathy; Mass Screening; Public Health.

\author{
'Departamento de Oftalmología. \\ Pontificia Universidad Católica de \\ Chile. Santiago, Chile. \\ ${ }^{2}$ Servicio de Oftalmología. \\ Hospital Dr. Sótero del Río. \\ Santiago, Chile. \\ ${ }^{\mathrm{a} P h D}$ en Epidemiología y \\ Bioestadística, University College \\ London, Inglaterra. \\ bBecado de Oftalmología. \\ Pontificia Universidad Católica de \\ Chile. Santiago, Chile. \\ La impresión de los formularios \\ fue financiada por la Fundación \\ de Salud Los Nogales. El trabajo \\ no recibió ningún otro tipo de \\ financiamiento. \\ Los autores declaran no tener \\ conflictos de interés. \\ Recibido el 21 de septiembre de \\ 2020, aceptado el 2 de marzo \\ de 2021. \\ Correspondencia a: \\ María Ibáñez Bruron \\ Departamento de Oftalmología, \\ Pontificia Universidad Católica \\ de Chile, Av. Diagonal Paraguay \\ 362, piso 3. \\ m.bruron@ucl.ac.uk
}

S egún la encuesta nacional de salud de Chile, más de $12 \%$ de la población adulta tiene diabetes, porcentaje que aumenta a $25 \%$ en personas con menor educación ${ }^{1}$. La retinopatía diabética (RD) es una complicación frecuente de la diabetes, siendo una de las primeras causas de discapacidad visual en personas en edad laboral ${ }^{2}$.
En Latinoamérica, hasta 40\% de los pacientes diabéticos tiene algún grado de $\mathrm{RD}$ y $17 \%$ requiere tratamiento ${ }^{3}$. Un centro de atención primaria en Chile reportó que $6 \%$ de los pacientes diabéticos tipo 2 tenía RD severa requiriendo tratamiento láser ${ }^{4}$. Entre los factores de riesgo de RD se encuentran la edad, duración de la diabetes, control 
glicémico, y comorbilidades cardiovasculares ${ }^{5}$. Importantemente, la historia de inasistencia al tamizaje es un predictor de lesiones severas que requieren derivación a oftalmólogo ${ }^{6}$.

El tamizaje de RD mediante fotografía digital ha logrado disminuir la incidencia de la discapacidad visual en aquellos países donde el diagnóstico oportuno y el tratamiento precoz de las lesiones detectadas están garantizados ${ }^{2,7}$. En Chile, la guía AUGE/GES de RD del Ministerio de Salud establece el examen anual de fondo de ojo con pupila dilatada por oftalmólogo a pacientes diabéticos tipo 1 a partir de los 12 años de edad o al $5^{\circ}$ año del diagnóstico y a los con diabetes tipo 2 a partir del diagnóstico ${ }^{8}$. Sin embargo, considerando información recolectada en el Servicio de Salud Metropolitano Sur Oriente (SSMSO) el año 2014, la cobertura del tamizaje solo llegaba a $21 \%{ }^{9}$. Similar a lo observado en otro estudio nacional ${ }^{10}$. La baja cobertura del tamizaje junto al aumento sostenido de diabetes en Chile y al envejecimiento poblacional, hacen imperativo la búsqueda de alternativas que aumenten el acceso y la cobertura del tamizaje.

En el sistema público de atención de salud chileno, las Unidades de Atención Primaria Oftalmológica (UAPOs) son responsables de realizar los controles oftalmológicos de pacientes con diabetes ${ }^{11}$. Tecnólogos médicos toman fotografías digitales de retina que luego son evaluadas y clasificadas por oftalmólogos mediante telemedicina ${ }^{12}$. Si bien las UAPOs facilitan el acceso a la toma de fotografía, esta estrategia de tamizaje aún depende de la disponibilidad de un oftalmólogo lo que dificulta su implementación y eleva sus $\operatorname{costos}^{13}$.

En el SSMSO, basado en programas de tamizaje de países desarrollados ${ }^{7,14}$, se ha implementado una estrategia de tamizaje en que tecnólogos médicos en oftalmología entrenados para este programa, realizan la toma de fotografías retinales, su evaluación inmediata y la derivación pertinente de los casos positivos a la confirmación diagnóstica y tratamiento por oftalmólogos 9 . Si bien la precisión de esta estrategia no ha sido evaluada ${ }^{9}$, la prevalencia y distribución de $\mathrm{RD}$ es similar a la reportada por estudios de tamizaje realizado por oftalmólogos por telemedicina ${ }^{10,15}$.

En el año 2018 se comenzó a implementar en algunos Servicios de Salud un sistema de clasificación inicial de las fotografías retinales mediante un software de inteligencia artificial llamado Diagnóstico Automatizado de Retinografías Telemáticas (DART) que clasifica las fotografías como alteradas o normales (positivas o negativas) ${ }^{16}$. Aquellas fotografías clasificadas inicialmente como alteradas por DART deben ser evaluadas por un oftalmólogo quien revisa las fotografías de forma telemática y decide la conducta a seguir de acuerdo al grado de RD observado en la fotografía. Esta estrategia de tamizaje, entonces, tiene dos etapas, un triage inicial (DART) que reduce la cantidad de fotografías a evaluar por el oftalmólogo a través de telemedicina, y una segunda etapa en la cual el oftalmólogo realiza el tamizaje propiamente $\operatorname{tal}^{16}$. El único artículo sobre DART reporta distintas precisiones a través del manuscrito, y no explicita los puntos de corte utilizados ${ }^{17}$.

El objetivo de este estudio fue estimar la exactitud diagnóstica de DART y de la estrategia utilizada en el SSMSO con tecnólogos médicos, para detectar RD y compararla con el diagnóstico establecido por oftalmólogos evaluando clínicamente a los participantes con lámpara de hendidura.

\section{Pacientes y Método}

Estudio observacional (no-experimental) de corte transversal que incluyó una muestra consecutiva de pacientes con diabetes que se realizaron el tamizaje de RD en el Centro de Diagnóstico y Tratamiento (CDT) del Hospital Dr. Sótero del Río (HSR) entre el 01 de julio y el 31 de agosto del 2019. El estudio se desarrolló de acuerdo con la Declaración de Helsinki y fue aprobado por el Comité de Ética del SSMSO. Los participantes firmaron un consentimiento informado.

El tamizaje de RD se realiza en el CDT del HSR en aquellos pacientes con diabetes de las comunas pertenecientes al SSMSO que no cuentan con UAPOs, pacientes que se controlan su diabetes en el nivel secundario de atención y pacientes que son derivados desde alguna UAPO de la red por una fotografía retinal con sospecha de RD. El tecnólogo médico no cuenta con las fotografías sospechosas en caso de ser pacientes derivados desde una UAPO. Todos los pacientes, independiente de su centro de referencia, son sometidos a la misma evaluación explicada más adelante.

A los participantes se les realizó una encuesta socio-demográfica y clínica. Luego se procedió a 
la toma de las fotografías con pupilas dilatadas (midriasis con tropicamida 1\% y fenilefrina 2,5\%) por tecnólogos médicos del CDT. Las fotografías fueron tomadas según las instrucciones del manual de DART que especifica la toma de al menos dos fotografías: una foto temporal, donde la papila quede lo más al costado posible y se alcance a ver completamente la emergencia de los vasos y una foto nasal, donde se sitúa la papila hacia el otro costado, pero dejando a lo menos un diámetro papilar desde el borde de la foto. El tecnólogo médico tuvo la libertad de tomar más fotografías si lo considerase necesario. Las fotos fueron tomadas usando el retinógrafo digital Polaroid $45^{\circ}$ (Canon CR2).

Después de tomadas las fotos, el tecnólogo médico evaluó y clasificó las fotografías de cada ojo usando el sistema simplificado elaborado por la Academia Americana de Oftalmología (AAO) ${ }^{18}$ en sin $\mathrm{RD}$, retinopatía diabética no proliferativa (RDNP) leve, RDNP moderada, RDNP severa y $\mathrm{RD}$ proliferativa. Luego, las fotografías fueron subidas a la plataforma DART para su evaluación centralizada. Si el tecnólogo medico tomó más de dos fotografías, éstas también fueron agregadas a la plataforma DART. Posteriormente, todos los participantes fueron evaluados por un oftalmólogo del HSR usando una lámpara de hendidura para examinar el fondo del ojo. El oftalmólogo se encontraba en una sala independiente, por lo que era ciego a la impresión diagnóstica del tecnólogo médico y de DART. Así mismo, ambos exámenes de tamizaje fueron ciegos al resultado de la confirmación diagnostica y al otro examen de tamizaje. Luego de la toma de fotografía los participantes debían permanecer en la sala de espera hasta ser llamados para su segunda evaluación. Aquellos participantes que se retiraron antes del examen de referencia fueron excluidos del análisis.

Se calculó la sensibilidad, y especificidad de cada examen de tamizaje con sus intervalos de confianza a $95 \%$. Se utilizó un solo ojo por participante (el peor ojo) para graduar la severidad de la RD. Se consideró como punto de corte de la RD casos con RDNP severa según oftalmólogo. Esta definición se basó en que son estos pacientes a quienes se les ofrece una intervención oftalmológica para cambiar el curso natural de la enfermedad. El punto de corte para considerar ambos examenes de tamizaje como positivo fue RDNP moderada. Se evaluó también un punto de corte más sensible (RDNP leve) pero solo para la estrategia del SSMSO ya que DART no entrega la información necesaria para calcular este punto de corte. DART tampoco revela el porcentaje de fotografías clasificadas como no evaluables. Las fotografías no evaluables para ambas estrategias fueron incluidas dentro de la definición de "tamizaje positivo".

\section{Resultados}

Durante el estudio, 510 participantes firmaron el consentimiento informado y contestaron la encuesta, de ellos 492 fueron evaluados por el TM y sus fotos subidas a la plataforma DART. El $75,4 \%(\mathrm{n}=371)$ de los participantes con fotografías, también fueron evaluadas con el estándar de referencia, el resto de los participantes no esperó la evaluación por el oftalmólogo por lo que fueron excluidos (Figura 1). No hubo diferencias significativas entre los participantes con o sin confirmación diagnóstica, excepto en la positividad de DART que fue menor en el grupo con confirmación diagnóstica (50\% versus $64 \%$, valor-p $=0,01$ ).

El promedio de edad de la muestra fue 61 años y $60,9 \%$ de ellos fueron mujeres (Tabla 1). La mayoría de los participantes pertenecían a consultorios de la comuna de La Granja $(\mathrm{n}=236$; $63,6 \%)$, comuna que deriva a todos los pacientes al HSR para su tamizaje de RD. El 11\% ( $\mathrm{n}=42)$ de los participantes eran controlados en el nivel secundario de atención. Solo 7,5\% de la muestra $(n=28)$ fueron pacientes derivados por fotografía retinal alterada. Respecto a la información clínica, 29,6\% de los participantes no sabía su tipo de diabetes. Entre los participantes que refirieron su tipo de diabetes, 77,4\% dijo tener diabetes tipo 2 . La mediana reportada de duración de la diabetes fue 6 años, con $58,4 \%$ de la muestra con una duración mayor de 5 años. Solo $17,5 \%(n=65)$ recordaba su última $\mathrm{HbA1c}$ con un promedio de 7,8\%.

La Figura 2 muestra la distribución de RD según severidad en el peor ojo. El 75,9\% $(\mathrm{n}=281)$ de la muestra no tenía signos de $\mathrm{RD}$ en ninguno de sus ojos mientras que $9,2 \%(\mathrm{n}=34)$ de la muestra tenía al menos signos de RDNP severa. Si se excluyen los participantes con RD panfotocoagulada, el porcentaje de pacientes con RDNP severa o RD proliferativa fue de $5,1 \%(18 / 354)$. 


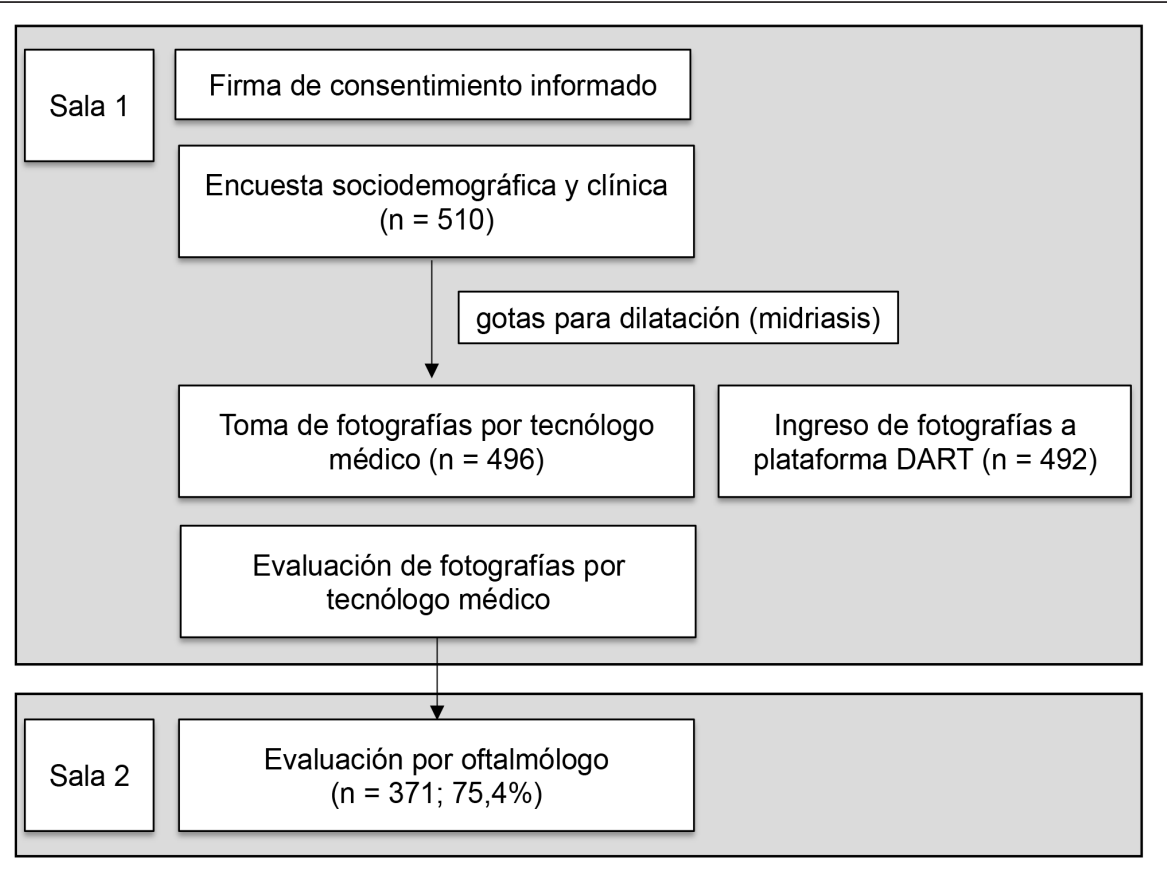

Figura 1. Flujograma de ingreso de los participantes.

\section{Tabla 1. Características socio-demográfica de la muestra con confirmación diagnóstica}

\begin{tabular}{|c|c|c|c|}
\hline \multicolumn{2}{|l|}{ Característica } & \multicolumn{2}{|c|}{ Participantes $(n=371)$} \\
\hline Mujeres, si & $n(\%)$ & 226 & $(60,9)$ \\
\hline Edad en años & promedio \pm de & 61,0 & $\pm 13,9$ \\
\hline \multicolumn{4}{|l|}{ Comuna de residencia } \\
\hline La Granja & $\mathrm{n}(\%)$ & 236 & $(63,6)$ \\
\hline Puente Alto & $\mathrm{n}(\%)$ & 69 & $(18,6)$ \\
\hline San José de Maipo & $\mathrm{n}(\%)$ & 23 & $(6,2)$ \\
\hline Pirque & $\mathrm{n}(\%)$ & 20 & $(5,4)$ \\
\hline La Florida & $\mathrm{n}(\%)$ & 9 & $(2,4)$ \\
\hline La Pintana & $\mathrm{n}(\%)$ & 7 & $(1,9)$ \\
\hline San Ramón & $\mathrm{n}(\%)$ & 7 & $(1,9)$ \\
\hline Duración diabetes en años $(n=353)$ & mediana [IQR] & 6,0 & {$[2,0-12,0]$} \\
\hline $\operatorname{HbA1c}(\%)(n=65)$ & promedio \pm de & 7,8 & $\pm 2,1$ \\
\hline Uso insulina, si & $\mathrm{n}(\%)$ & 111 & $(29,9)$ \\
\hline Hipertensión, si & $\mathrm{n}(\%)$ & 246 & $(66,3)$ \\
\hline Dislipidemia, si & $n(\%)$ & 198 & $(53,4)$ \\
\hline Tabaco, si & $n(\%)$ & 82 & $(22,1)$ \\
\hline \multicolumn{4}{|l|}{ Complicaciones crónicas diabetes } \\
\hline Diálisis & $\mathrm{n}(\%)$ & 13 & $(3,5)$ \\
\hline Amputación & $\mathrm{n}(\%)$ & 9 & $(2,4)$ \\
\hline Retinopatía tratada con láser & $\mathrm{n}(\%)$ & 11 & $(3,0)$ \\
\hline
\end{tabular}

de: desviación estándar; IQR: rango intercuartil. 


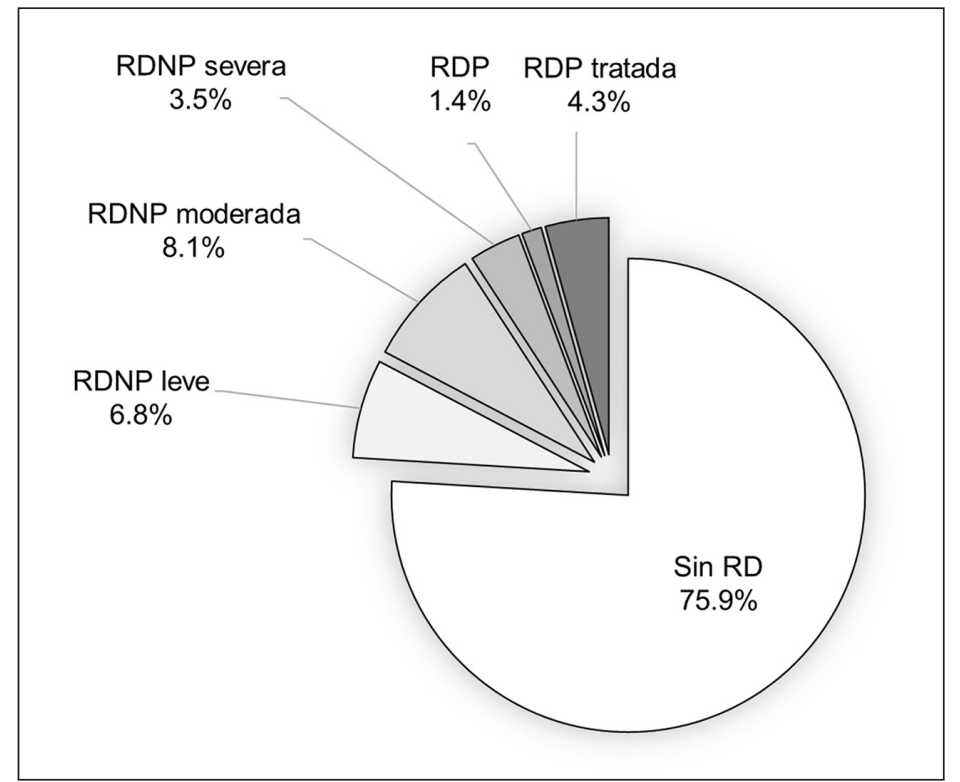

Figura 2. Distribución de retinopatía diabética según evaluación por oftalmólogo ( $n=370$, un participante con opacidad de medios bilateral no pudo ser clasificado). RD: retinopatía diabética; RDNP: retinopatía diabética no proliferativa; RDP: retinopatía diabética proliferativa.
De los 371 participantes con confirmación diagnóstica, DART clasificó a 185 participantes (49,9\%) como alterados en su evaluación automatizada. Estos 185 participantes se consideraron positivos para DART. Las fotografías de estos pacientes requerían una segunda evaluación por oftalmólogo por telemedicina para decidir la pertinencia de su derivación a confirmación diagnóstica. Sin embargo, estas evaluaciones no estuvieron disponibles hasta la escritura de este manuscrito, que fue 11 meses después. Por su parte, los tecnólogos médicos clasificaron como positivos a $62(16,7 \%)$ participantes incluyendo $47(12,7 \%)$ participantes con al menos RDNP moderada en un ojo y $15(4,0 \%)$ participantes con fotografías no evaluables.

La Tabla 2 muestra la clasificación de los participantes según el oftalmólogo y ambos exámenes de tamizaje utilizando como punto de corte RDNP moderada. DART mostró una sensibilidad de 100\% (IC95\%: 90-100\%) y una especificidad de 55,4\% (IC95\%: 50-61\%) para detectar casos con al menos RDNP severa. Los tecnólogos médicos tuvieron una sensibilidad de 97,1\% (IC95\%: 85$100 \%$ ) y una especificidad de $91,7 \%$ (IC95\%: 88 94\%) para detectar estos casos. El valor predictivo positivo de DART y los tecnólogos médicos fue de $18,5 \%$ y $54,1 \%$, respectivamente. El valor predicti-

Tabla 2. Rendimiento de exámenes de tamizaje para detectar RDNP severa o casos más graves según oftalmólogo

\begin{tabular}{|c|c|c|c|}
\hline \multicolumn{2}{|c|}{$\begin{array}{l}\text { Examen de tamizaje (RDNP moderada o } \\
\text { peor + fotografía no evaluable) }\end{array}$} & \multicolumn{2}{|c|}{$\begin{array}{l}\text { Examen de referencia: oftalmólogo } \\
\text { (RDNP severa o peor) } n=370^{*}\end{array}$} \\
\hline & & Positivo & Negativo \\
\hline \multirow[t]{2}{*}{ DART } & Positivo & 34 & 150 \\
\hline & Negativo & 0 & 186 \\
\hline \multirow[t]{2}{*}{ Tecnólogo médico } & Positivo & 33 & 28 \\
\hline & Negativo & 1 & 308 \\
\hline
\end{tabular}

*1 paciente fue clasificado como no evaluable en ambos ojos por el oftalmólogo ( $n=370)$. RDNP: retinopatía diabética no proliferativa. 
vo negativo de DART y los tecnólogos médicos fue de $100 \%$ y $99,7 \%$, respectivamente. El único caso de RD no identificado por los tecnólogos médicos fue un participante con una $\mathrm{RD}$ panfotocoagulada unilateral. Considerando un punto de corte más sensible para los tecnólogos médicos (al menos RDNP leve), su sensibilidad aumentó a 100\% (IC95\%: 90-100\%) y su especificidad disminuyó a 74,4\% (IC95\%: 69-79\%) para detectar casos de al menos RDNP severa.

\section{Discusión}

El uso de inteligencia artificial para automatizar el tamizaje de RD ha surgido como una estrategia para disminuir los costos y aumentar el acceso de los pacientes a los programas de tamizaje ${ }^{19}$. Sin embargo, como cualquier intervención, debe asegurar su seguridad, efectividad y reproducibilidad antes de ser utilizada en pacientes ${ }^{20}$. El objetivo de este estudio fue comparar los resultados de dos estrategias de tamizaje de RD actualmente utilizadas en el sistema público de salud en Chile, una de las cuales utiliza inteligencia artificial.

Las estrategias evaluadas en este estudio demostraron una alta sensibilidad para identificar casos de al menos RDNP severa. Sin embargo, la especificidad del tecnólogo medico fue significativamente superior a DART ( $92 \%$ vs. $55 \%$ ), incluso cuando se utilizó el punto de corte de positividad más sensible (y menos especifico) del tecnólogo medico (74\% vs. 55\%). La baja especificidad de DART puede ser explicada por la ausencia de la segunda evaluación de las fotografías por oftalmólogos a través de telemedicina. Esta segunda evaluación podría haber disminuido el número de casos positivos que requerían confirmación diagnóstica, lo que mejoraría su especificidad. Sin embargo, este segundo filtro de derivación pudiera haber generado también una disminución de la sensibilidad de la estrategia. La demora en la entrega de resultados al igual que la falta de especificidad de DART -que se traduce en un alto número de falsos positivos - puede tener un impacto psicológico deletéreo en las personas disminuyendo la adherencia al tamizaje.

Una ventaja de la estrategia DART es que reduciría a 50,4\% la carga de los oftalmólogos como tamizadores por telemedicina. Es decir, tendrían que mirar la mitad de las fotografías de fondo de ojo. Sin embargo, aún depende fuertemente de ellos. Considerando cifras del censo $2017^{21}$, cada año habría más de 1,7 millones de personas de 15 años o más que requieren tamizaje de RD. Reducir la evaluación por oftalmólogos a 850.000 pacientes, si bien significativa, no es suficiente. Actualmente, existen otras estrategias de inteligencia artificial con mejor rendimiento que el demostrado por $\mathrm{DART}^{22}$, que podrían reducir la necesidad de evaluación oftalmológica sin transar la detección de casos de RD.

El sistema actualmente implementado en el HSR, no depende de oftalmólogos como tamizadores, entrega resultados inmediatos y deriva solo a 16,7\% de los participantes a confirmación diagnostica. A diferencia de DART, no requiere que el tecnólogo medico traspase las fotografías desde la cámara retinal a un computador para luego subir las fotos a una plataforma, lo cual disminuye la eficiencia del tamizaje. El entrenamiento recibido por los tecnólogos médicos que clasifican las fotografías en el SSMSO es mayor al requerido por los tecnólogos médicos que solo toman fotografías en la estrategia DART. Sin embargo, es probable que el rendimiento de DART sea replicable por tecnólogos médicos con menor entrenamiento que los que realizan el tamizaje en el HSR.

Una limitación de este estudio fue que 24,6\% de la muestra se retiró del hospital antes de realizarse la confirmación diagnóstica debiendo ser excluidos. Esta limitación pudo incidir en la estimación de la prevalencia de la RD en esta muestra, pero no en la estimación de la sensibilidad y especificidad ya que éstas son independientes a la prevalencia de la enfermedad.

La selección de un examen de tamizaje no solo depende de su capacidad de diferenciar población sana de la posiblemente enferma, sino que debe considerar el contexto donde se implementará, estando inserto en un programa que considere los principios básicos del tamizaje ${ }^{7}$. Un programa de tamizaje debe tener un objetivo y una población clara (qué queremos detectar y en quiénes), debe garantizar el acceso universal al examen de tamizaje, confirmación diagnóstica y tratamiento, y debe monitorizar periódicamente la calidad y el cumplimiento de metas para asegurar su costo-efectividad. Nos unimos al llamado de levantar datos locales acerca de la prevalencia de RD y cobertura del tamizaje ${ }^{23}$, para informar el desarrollo de un programa de tamizaje siguiendo las 
recomendaciones de la Asociación Panamericana de Oftalmología ${ }^{3}$. En la creación de este programa deben participar pacientes y personal de salud de todos los niveles de atención de salud reflejando el cuidado multidisciplinario que requieren las personas con diabetes.

Agradecimientos: Se agradece la cooperación del equipo de oftalmología del HSR, tanto tecnólogos médicos como Técnicos en Enfermería Nivel Superior (TENS) y médicos.

\section{Referencias}

1. Ministerio de Salud de Chile. Encuesta Nacional de salud (ENS): primeros resultados 2016-2017. https:// www.minsal.cl/wp-content/uploads/2017/11/ENS2016-17_PRIMEROS-RESULTADOS.pdf (acceso agosto 2020).

2. Liew G, Michaelides M, Bunce C. A comparison of the causes of blindness certifications in England and Wales in working age adults (16-64 years), 1999-2000 with 2009-2010. BMJ Open. 2014; 4 (2): e004015.

3. PAAO. 2016. Diabetic Retinopathy Guidelines - ICO PAAO 2016. https://www.iapb.org/resources/diabetic-retinopathy-guidelines-ico-paao-2016-spanish/ (acceso agosto 2020).

4. Abuauad S, Guzmán P, Urzúa C. Prevalencia de retinopatía diabética y edema macular en población diabética del CESFAM Cordillera Andina de Los Andes. Rev Chilena Salud Pública 2014; 18 (1) 81-6. doi: 10.5354/07195281.2014.30759.

5. Yau JW, Rogers SL, Kawasaki R, Lamoureux EL, Kowalski JW, Bek T, et al. Global prevalence and major risk factors of diabetic retinopathy. Diabetes Care. 2012; 35 (3): 556-64.

6. Scanlon PH, Aldington SJ, Stratton IM. Delay in diabetic retinopathy screening increases the rate of detection of referable diabetic retinopathy. Diabet Med 2014; 31 (4): 439-42.

7. Scanlon PH. The English National Screening Programme for diabetic retinopathy 2003-2016. Acta diabetologica. 2017; 54 (6): 515-25.

8. Ministerio de Salud de Chile. Guía clínica: Retinopatía diabética. 2010. http://www.minsal.cl/portal/url/ item/7222754637ab8646e04001011f014e64.pdf (acceso agosto 2020).

9. Covarrubias T, Delgado I, Rojas D, Coria M. Tamizaje en el diagnóstico y prevalencia de retinopatía diabética en atención primaria. Rev Med Chile 2017; 145 (5): 564-

\section{1. doi:10.4067/S0034-98872017000500002.}

10. Avendaño-Veloso A, Parada-Hernández F, González-Ramos R, Dougnac-Osses C, Carrasco-Sáez JL, Scanlon PH. Teleophthalmology: a strategy for timely diagnosis of sight-threatening diabetic retinopathy in primary care, Concepción, Chile. Int J Ophthalmol. 2019; 12 (9): 1474-8. doi: 10.18240/ijo.2019.09.16.

11. Riesco B, Sáez V, Escobar S, Barría F, Donoso R, Gil C. Unidades de atención primaria en oftalmología en Chile: historia y funciones. Rev Med Chile 2015; 143 (7): 919-24. doi:10.4067/S0034-98872015000700013.

12. González-Ramos R, Cadegan-Segura B, Avendaño-Veloso A, Parada-Hernández F, Cabezas-Monsalve A. Teleoftalmología como apoyo a la atención primaria en salud en la pesquisa de la retinopatía diabética. 2014. Poster presentado en el I Congreso Iberoamericano de Telesalud y Telemedicina. Lima, Perú.

13. Nguyen HV, Tan GS, Tapp RJ, Mital S, Ting DS, Wong HT, et al. Cost-effectiveness of a National Telemedicine Diabetic Retinopathy Screening Program in Singapore. Ophthalmology. 2016; 123 (12): 2571-80.

14. Peto T, Tadros C. Screening for diabetic retinopathy and diabetic macular edema in the United Kingdom. Curr Diab Rep. 2012; 12 (4): 338-45.

15. Flores R, Donoso R, Anguita R. Modelo de manejo en red y por telemedicina de la retinopatía diabética en dos comunas del Servicio de Salud Metropolitano Oriente. Rev Med Chile 2019; 147 (4): 444-50. doi: 10.4067/ S0034-98872019000400444.

16. Ministerio de Salud de Chile. Ministro de Salud presenta software que permitirá triplicar la cantidad de exámenes para prevenir la ceguera diabética https://www.minsal. $\mathrm{cl} /$ ministro-de-salud-presenta-software-que-permitira-triplicar-la-cantidad-de-examenes-para-prevenir-la-ceguera-diabetica/ (acceso agosto 2020).

17. Arenas-Cavalli JT, Ríos SA, Pola M, Donoso R. A web-based platform for automated diabetic retinopathy screening. Procedia Comput Sci. 2015; 60: 557-63.

18. Wilkinson CP, Ferris FL 3rd, Klein RE, Lee PP, Agardh CD, Davis M, et al; Global Diabetic Retinopathy Project Group. Proposed international clinical diabetic retinopathy and diabetic macular edema disease severity scales. Ophthalmology. 2003; 110 (9): 1677-82. doi:10.1016/S0161-6420(03)00475-5.

19. Vujosevic S, Aldington SJ, Silva P, Hernández C, Scanlon P, Peto T, et al. Screening for diabetic retinopathy: new perspectives and challenges. Lancet Diabetes Endocrinol. 2020; 8 (4): 337-47. doi: 10.1016/S22138587(19)30411-5.

20. Vollmer S, Mateen BA, Bohner G, Király FJ, Ghani R, Jonsson P, et al. Machine learning and artificial intelli- 
gence research for patient benefit: 20 critical questions on transparency, replicability, ethics, and effectiveness. BMJ. 2020;368:16927. doi: 10.1136/bmj.16927.

21. Instituto Nacional de Estadísticas (INE). Resultados del Censo 2017. https://www.ine.cl/estadisticas/sociales/ censos-de-poblacion-y-vivienda/poblacion-y-vivienda (acceso agosto 2020).

22. Raman R, Srinivasan S, Virmani S, Sivaprasad S, Rao
C, Rajalakshmi R. Fundus photograph-based deep learning algorithms in detecting diabetic retinopathy. Eye (Lond). 2019; 33 (1): 97-109. doi: 10.1038/s41433018-0269-y.

23. Covarrubias T, Oyarte M, Cabieses B, Coria M. Chile necesita mejores estadísticas y mayor cobertura en tamizaje de retinopatía diabética. Rev Med Chile 2017; 145 (12): 1633-5. doi: 10.4067/s0034-98872017001201633. 